\title{
Evidence for an inner molecular disk around massive Young Stellar Objects ${ }^{\star}$
}

\author{
A. Bik and W. F. Thi \\ Astronomical Institute "Anton Pannekoek", University of Amsterdam, Kruislaan 403, 1098 SJ Amsterdam, The Netherlands \\ e-mail: bik@science.uva.nl \\ Received 27 May 2004 / Accepted 2 October 2004

\begin{abstract}
We present observations of $\mathrm{CO}$ overtone bandhead emission toward four massive Young Stellar Objects (spectral type O6-B5). The high signal-to-noise ratio $K$-band spectra were obtained with VLT-ISAAC at a resolution $\Delta v=30 \mathrm{~km} \mathrm{~s}^{-1}$, sufficient to resolve the bandheads, but not the individual $J$-lines. We are able to explain the shape of the lines by assuming a simple isothermal Keplerian disk model seen at different inclinations. The gas temperature ranges from 1500 to $4500 \mathrm{~K}$ and the $\mathrm{CO}$ column density is between 0.1 and $4 \times 10^{21} \mathrm{~cm}^{-2}$. The emission probably arises within the first few astronomical units of the disk, consistent with the high gas temperature. Our results indicate that molecules can survive close to a hot star and suggest
\end{abstract} \\ that dense $\left(n_{\mathrm{H}}>10^{10} \mathrm{~cm}^{-3}\right)$ inner disks may be relatively common at an advanced stage of high-mass star formation.
}

Key words. stars: early-type, formation - circumstellar matter - molecular processes - line: profiles

\section{Introduction}

Disks are found around many young low-mass stars as a natural byproduct of angular momentum conservation during the star formation process. Observational evidence for disks around their more massive counterparts remains sparse, although they may be essential during the formation of highmass stars (Churchwell 2002). Extended disks have been found around massive young stellar objects (YSOs) in the Hot Core phase (e.g. Shepherd et al. 2001; Beltrán et al. 2004; Garay \& Lizano 1999; Chini et al. 2004), albeit those millimeter observations solely probe gas beyond $1000 \mathrm{AU}$. In the later Herbig Be stage, disks have only been recently discovered (Fuente et al. 2003).

Evidence for the presence of a gaseous disk around the early B star NGC 2024-IRS2 is presented by Lenorzer et al. (2004). They argue that the infrared excess observed toward NGC 2024-IRS2 is best explained by the emission from a dense gaseous disk as close as $0.3 \mathrm{AU}$ to the star.

At the high densities and temperatures (1000-2000 K) characteristic of disks at 0.1-5 AU around young stars, molecules are expected to be sufficiently excited to produce a rich ro-vibrational spectrum in the near- (overtone transitions $\Delta v= \pm 2$ ) and mid-infrared (fundamental transitions $\Delta v= \pm 1$ ). $\mathrm{CO}$ first overtone emission is most likely emitted by a disk and/or a wind and has been detected and analyzed by, e.g., Scoville et al. (1979), Geballe \& Persson (1987), Carr (1989),

* Based on observations collected at the European Southern Observatory at La Silla and Paranal, Chile (ESO Programmes 68.C-0652 and 69.C-0448)
Chandler et al. $(1993,1995)$ toward a number of other highand low-mass stars. Their studies were, however, hampered by the small number of observed $J$-lines or a low resolving power and moderate signal-to-noise.

Bik et al. (2004) examine $K$-band spectra of a sample of massive YSOs associated with ultra-compact HII regions (UCHII). Here, we present a simple disk model that describes the $\mathrm{CO}$ bandhead emission detected in four of these objects. The observations are presented in Sect. 2. The disk model and the results are shown in Sect. 3. In Sect. 4 we discuss the location and origin of the $\mathrm{CO}$. The implications of our findings on the process of high-mass star formation are summarized in Sect. 5 .

\section{Observations}

High signal-to-noise ratio long-slit $K$-band spectra of four massive YSOs have been obtained in 2002 with the $I S A A C$ spectrometer mounted on the VLT. A narrow slit $\left(0.3^{\prime \prime}\right)$ is used, resulting in a resolving power of $R=10000(\Delta v=$ $30 \mathrm{~km} \mathrm{~s}^{-1}$ ). The spectra were reduced using standard procedures. The wavelength calibration is based on the telluric $\mathrm{OH}$ emission line spectra. Telluric standard stars of spectral type $\mathrm{A} 0 \mathrm{~V}$, observed under identical sky conditions as the science targets, were used to correct for the telluric absorption lines. A detailed description and analysis of the $K$-band spectra can be found in Bik et al. (2004). The properties of the objects are summarized in Table 1. 


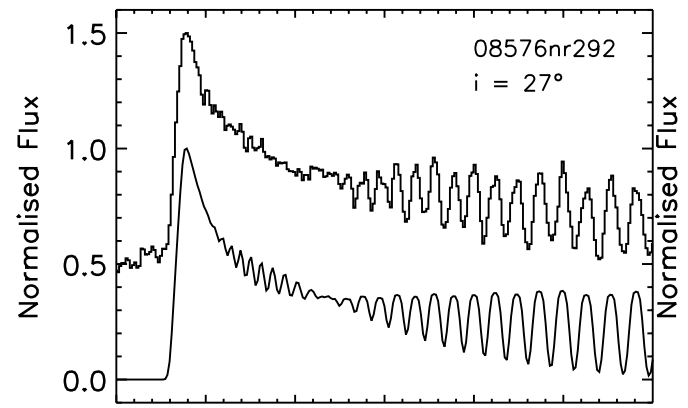

2.2902 .2952 .3002 .3052 .3102 .315 wavelength (micron)

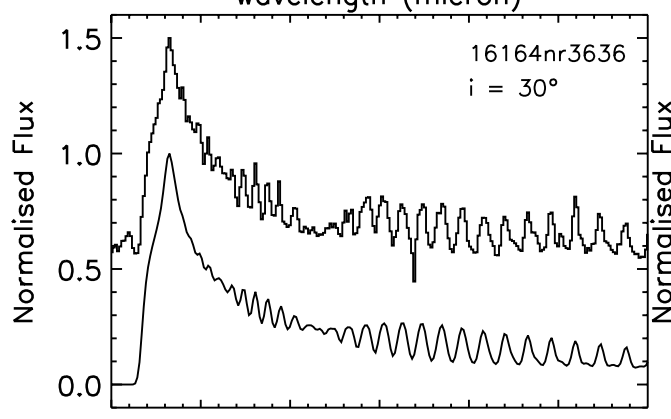

2.2902 .2952 .3002 .3052 .3102 .315 wavelength (micron)

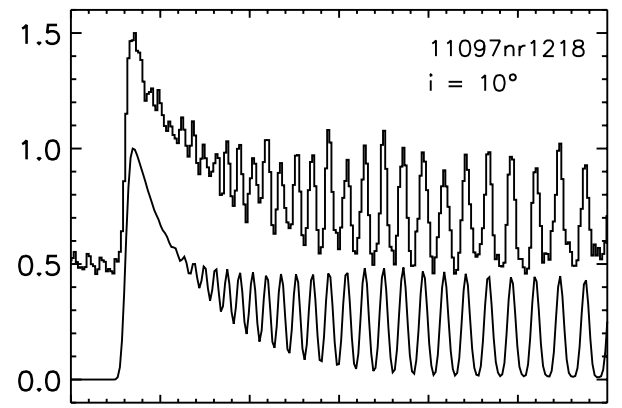

2.2902 .2952 .3002 .3052 .3102 .315 wavelength (micron)

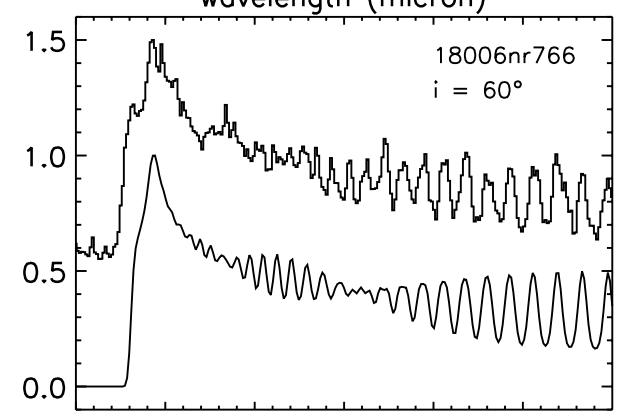

2.2902 .2952 .3002 .3052 .3102 .315 wavelength (micron)

Fig. 1. $K$-band observed (upper spectra) and modeled (lower spectra) spectra of the CO 2-0 bandhead. The name of the object and the derived inclination is displayed. By definition $0^{\circ}$ inclination corresponds to a disk seen pole-on.

\section{Disk model}

Continuum subtracted and normalized first-overtone bandhead spectra are shown in Fig. 1 (upper spectra). Four overtones are detected but only the first one is displayed. The shape of the bandhead varies from source to source. The rise of the first bandhead is sharp in the spectrum of 08576nr292 and $11097 \mathrm{nr} 1218$ whereas in that of $16164 \mathrm{nr} 3636$ and $18006 \mathrm{nr} 766$ a blue wing is clearly present.

The expected profile from a spherical stellar wind has a flattopped shape and cannot account for the wing seen in two of the objects. The favored model to explain the blue wing consists of a Keplerian disk and/or a disk-wind (e.g. Chandler et al. 1995), although the disk model provided the best match. In this paper, we explore the possibility that the $\mathrm{CO}$ bandhead emission arises solely from a small disk in Keplerian rotation around the massive star. We generate synthetic CO bandhead spectra using a standard parametric disk model for a range of gas temperatures, column densities, turbulent velocities and disk viewing angles (cf. Kraus et al. 2000, for a detailed description). In contrast to standard disk models, the disk is assumed to be entirely isothermal. The molecular data (line frequencies and Einstein coefficients) are taken from the database of Chandra et al. (1996). At temperatures above $1500 \mathrm{~K}$, needed to populate the higher vibrational levels, no dust grains can survive and, therefore, no dust opacity is included. The population of the $\mathrm{CO}$ rotational levels within each vibrational level is assumed to be in local thermodynamic equilibrium (LTE), at the same temperature $T_{\mathrm{ex}}$ as the local vibrational temperature. Each bandhead is characterized by a specific vibrational temperature. The downhill simplex method is used to determine the model parameters: the $\mathrm{CO}$ total column density $N(\mathrm{CO})$, the excitation temperature $T_{\mathrm{ex}}$, the disk inner and outer radius $R_{\min }$ and $R_{\max }$ (Table 1 ) corresponding to the best fitting model for each object (see the bottom spectra in each panel of Figs. 1 and 2). The stellar mass and the inclination are degenerate parameters. The parameters are best constrained by the first bandhead which is not contaminated by the $P$-branch of the other bandheads nor by Hydrogen Pfund lines. For 11097nr1218, we were able to fit the four bandheads (Fig. 2) which shows that $T_{\mathrm{ex}}$ is lower for higher $v$ (see next section).

\section{Results and discussion}

We have observed the $\mathrm{CO}$ bandheads in a sample of 15 massive YSOs and five show these lines in emission (Bik et al. 2004). In this letter, four of these objects are modeled and are well described by optically thin CO gas located in a Keplerian disk. From the quality of the fits, it is clear that additional emission from a disk-wind is not required.

Our results show that the $\mathrm{CO}$ gas is located within a few $\mathrm{AU}$ of the central star (Table 1). The maximum distance where the $\mathrm{CO}$ bandheads are emitted depends on the inclination, which is not a well constrained parameter, but even for a wide range of possible inclinations the hot $\mathrm{CO}$ molecules are located within a few AU from the star.

In the absence of extinction by dust grains, the $\mathrm{CO}$ molecules should be photodissociated by the stellar ultraviolet photons. However, the derived column densities $\left(10^{20}-10^{21} \mathrm{~cm}^{-2}\right)$ are well above the required value for the CO molecules to self-shield $\left(N(\mathrm{CO}) \sim 10^{15} \mathrm{~cm}^{-2}\right.$, van Dishoeck $\&$ Black 1988). Moreover, gas phase chemical models show 


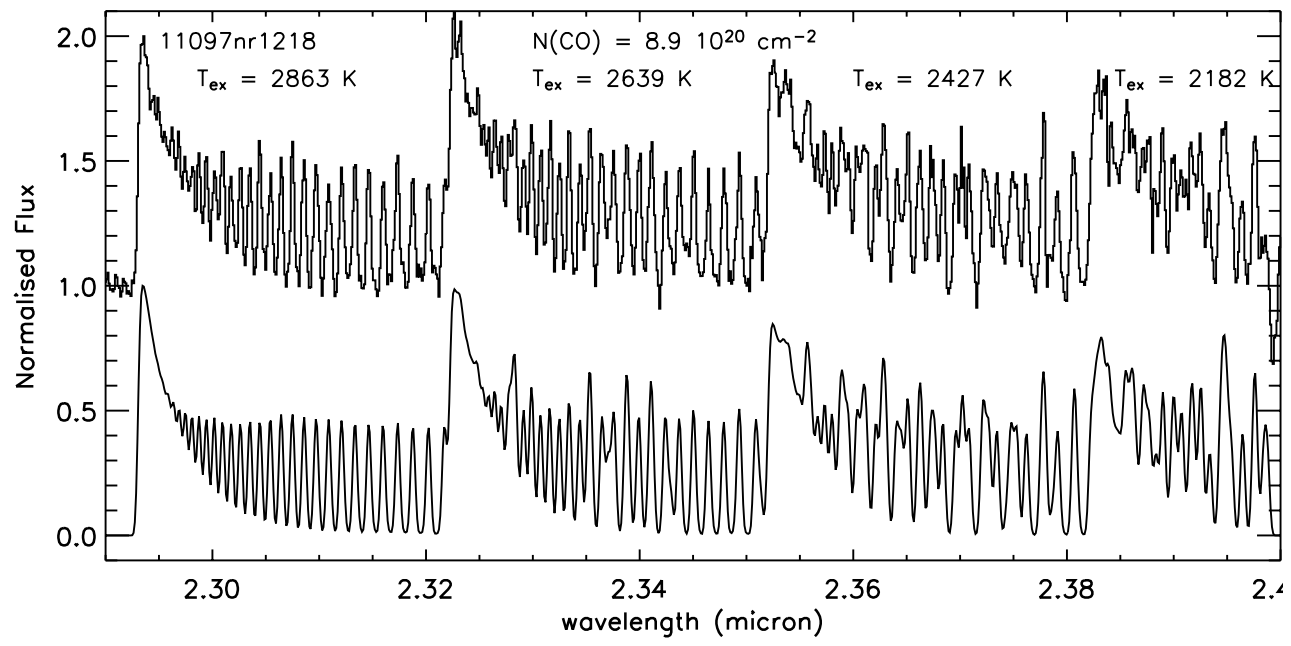

Fig. 2. Fit of the complete observed CO bandhead spectrum of 11097nr1218. The upper curve is the observed spectrum whereas the lower one is the model spectrum.

Table 1. Stellar properties and fit parameters of the first $(v=2-0)$ bandhead. The masses of the objects (Col. 3) are based on spectral type estimates, determined from their position in the $(K, J-K)$ color-magnitude diagram (Bik et al. 2004). The reference of the distances (Col. 4) are given in Bik et al. (2004). The errors on the inclination reflect the range of inclinations where a good fit was possible. The errors on $R_{\min }$ and $R_{\max }$ are the corresponding values for these inclinations. The errors on $T_{\text {ex }}$ and $N(\mathrm{CO})$ are dominated by systematic instead of statistical errors.

\begin{tabular}{|c|c|c|c|c|c|c|c|c|c|c|}
\hline IRAS & Object & $\begin{array}{l}\text { Mass } \\
\left(M_{\odot}\right)\end{array}$ & $\begin{array}{c}d \\
(\mathrm{kpc})\end{array}$ & $\begin{array}{l}T_{\mathrm{ex}} \\
(\mathrm{K})\end{array}$ & $\begin{array}{l}N(\mathrm{CO}) \\
\left(\mathrm{cm}^{-2}\right)\end{array}$ & $\begin{array}{c}i \\
\left({ }^{\circ}\right)\end{array}$ & $\begin{array}{l}R_{\min } \\
(\mathrm{AU})\end{array}$ & $\begin{array}{l}R_{\max } \\
(\mathrm{AU})\end{array}$ & $\begin{array}{c}M(\mathrm{CO}) \\
\left(M_{\odot}\right)\end{array}$ & $\overline{\chi_{v}^{2}}$ \\
\hline $16164-5046$ & $16164 \mathrm{nr} 3636$ & 30 & 3.6 & 4480 & $3.9 \times 10^{20}$ & $30_{-23}^{+6}$ & $3.1_{-2.9}^{+18.2}$ & $3.2_{-3.0}^{+123.5}$ & $2.0 \times 10^{-9}$ & 0.99 \\
\hline $11097-6102$ & 11097nr1218 & 11 & 2.8 & 2710 & $8.9 \times 10^{20}$ & $10_{-5}^{+10}$ & $0.6_{-0.4}^{+0.1}$ & $3.3_{-3.1}^{+13.5}$ & $2.6 \times 10^{-7}$ & 1.51 \\
\hline $18006-2422$ & $18006 \mathrm{nr} 766$ & 11 & 1.9 & 1800 & $1.0 \times 10^{21}$ & $60_{-30}^{+20}$ & $0.2_{-0.06}^{+0.24}$ & $1.9_{-16}^{+2.5}$ & $6.2 \times 10^{-8}$ & 1.72 \\
\hline 08576-4334 & 08576nr292 & 6 & 0.7 & 1660 & $3.9 \times 10^{21}$ & $27_{-14}^{+2}$ & $0.2_{-0.1}^{+1.1}$ & $3.6_{-3.4}^{+70}$ & $8.4 \times 10^{-7}$ & 2.01 \\
\hline
\end{tabular}

that $\mathrm{CO}$ molecules can rapidly form in the gas phase to compensate for their destruction.

The CO column densities in the four objects only differ by at most a factor of 10 . This reflects the narrow parameter range in density and temperature at which the $\mathrm{CO}$ first-overtone bandheads are emitted. A steep density and/or temperature gradient can result in a very narrow region in the disk where the $\mathrm{CO}$ firstovertone is emitted. This is reflected in the value of $R_{\min }$ and $R_{\max }$ in e.g. $16164 \mathrm{nr} 3636$, where only a narrow ring in the possibly much more extended disk is producing the $\mathrm{CO}$ bandheads.

The derived excitation temperatures range from 1700 to $4500 \mathrm{~K}$. This temperature may not be equal to the gas kinetic temperature for the high- $v$ transitions since the critical density is increasing with $v\left(n_{\mathrm{H}}>10^{10} \mathrm{~cm}^{-3}\right)$. In addition, the fit to all four bandheads shows that $T_{\mathrm{ex}}$ is lower for higher $v$ transitions. Therefore, the population of the high $v$ levels is likely sub-thermal. The excitation temperature shows a positive trend with increasing effective temperature of the central star (Table 1). One possibility is that the diluted near-infrared radiation field pumps the higher $v$-levels. Likewise, UV fluorescence will advantageously populate the high $v$-levels. Alternatively, the earlier type stars have higher luminosity which results in an increased heating by photo-electrons. The later explanation is favored because both radiation pumping mechanisms (UV and near-IR) will preferably populate the higher rather than the lower $v$-levels, which is not corroborated by the data. However, only a detailed model that includes all the CO levels and possible pumping mechanisms will give a definitive answer.

Two scenarios can explain the presence of an inner disk at the stage of massive star formation where the circumstellar environment is being dispersed by the stellar wind and radiation.

According to the first scenario, the disk material may originate from the rapidly rotating star itself. However, only the wind of a $\mathrm{B}[\mathrm{e}]$ supergiant can produce a disk with sufficiently high densities to emit CO bandheads (McGregor et al. 1988; Kraus \& Lamers 2003). The stellar characteristics exclude the possibility that they are supergiants (Bik et al. 2004).

Alternatively, the inner disk may be the remnant of a massive and extended (up to $10000 \mathrm{AU}$ ) accretion disk detected in the early phase of star formation (e.g. Shepherd et al. 2001; Beltrán et al. 2004; Garay \& Lizano 1999; Chini et al. 2004). In the model of disk dispersal by Hollenbach et al. (1994), the photoevaporation of the outer part is faster than the dispersion of the inner region by stellar winds. This model naturally explains both the presence of inner disks and the absence of large outer disks in most Herbig Be stars (e.g. Natta et al. 2000; Fuente et al. 2003). 


\section{Conclusions and prospects}

We have modeled the CO first-overtone bandhead emissions of 4 massive YSOs. The different profiles of the $\mathrm{CO}$ bandheads are all well explained by a simple Keplerian disk. Regardless of the gas excitation mechanism, the information on the gas kinematics given by the shape of the $\mathrm{CO}$ bandheads shows that the $\mathrm{CO}$ is located close to the star (0.1-5 AU).

The $\mathrm{CO}$ overtone emission likely arises in a narrow torus where the gas temperature is between 1500 and $4500 \mathrm{~K}$. This range of temperature reflects the difference in effective temperature of the central objects. The decrease in excitation temperature for one object from the $v=2-0$ bandhead to the $v=5-3$ bandhead indicates that the higher $v$-levels are subthermally populated. Excitation mechanisms other than LTE, like UV or infrared pumping and photo-electric heating cannot be excluded by the simple model used here.

The detected disks may be the remnants of massive accretion disks. In particular, it is the first evidence of a disk around a young O6-O8 star, suggesting that these objects are recently formed as the disks are not yet dispersed by the stellar FUV photons and stellar wind.

Spatially resolved observation of CO bandhead emissions with Amber at the VLT interferometer will provide further constraints on the size and the geometry of the $\mathrm{CO}$ emitting region.

Acknowledgements. W.F.T. is supported by NWO grant 614.041.005. The authors thank Rens Waters, Lex Kaper and Doug Johnstone for fruitful discussion and the VLT staff for support and help with the observations.

\section{References}

Beltrán, M. T., Cesaroni, R., Neri, R., et al. 2004, ApJ, 601, L187

Bik, A., Kaper, L., Waters, L. B. F. M., \& Hanson, M. M. 2004, A\&A, submitted

Carr, J. S. 1989, ApJ, 345, 522

Chandler, C. J., Carlstrom, J. E., \& Scoville, N. Z. 1995, ApJ, 446, 793

Chandler, C. J., Carlstrom, J. E., Scoville, N. Z., Dent, W. R. F., \& Geballe, T. R. 1993, ApJ, 412, L71

Chandra, S., Maheshwari, V. U., \& Sharma, A. K. 1996, A\&AS, 117, 557

Chini, R., Hoffmeister, V., Kimeswenger, S., et al. 2004, Nature, 429, 155

Churchwell, E. 2002, ARA\&A, 40, 27

Fuente, A., Rodríguez-Franco, A., Testi, L., et al. 2003, ApJ, 598, L39

Garay, G., \& Lizano, S. 1999, PASP, 111, 1049

Geballe, T. R., \& Persson, S. E. 1987, ApJ, 312, 297

Hollenbach, D., Johnstone, D., Lizano, S., \& Shu, F. 1994, ApJ, 428, 654

Kraus, M., \& Lamers, H. J. G. L. M. 2003, A\&A, 405, 165

Kraus, M., Krügel, E., Thum, C., \& Geballe, T. R. 2000, A\&A, 362, 158

Lenorzer, A., Bik, A., de Koter, A., et al. 2004, A\&A, 414, 245

McGregor, P. J., Hyland, A. R., \& Hillier, D. J. 1988, ApJ, 324, 1071

Natta, A., Grinin, V., \& Mannings, V. 2000, Protostars and Planets IV, 559

Scoville, N. Z., Hall, D. N. B., Ridgway, S. T., \& Kleinmann, S. G. 1979, ApJ, 232, L121

Shepherd, D. S., Claussen, M. J., \& Kurtz, S. E. 2001, Science, 292, 1513

van Dishoeck, E. F., \& Black, J. H. 1988, ApJ, 334, 771 